\title{
Predicting military and veteran suicide risk: Cultural aspects
}

\author{
Paul Thompson \\ Dartmouth College \\ Paul.Thompson@dartmouth.edu
}

\author{
Chris Poulin \\ Durkheim Project \\ chris@durkheimproject.org
}

\author{
Craig J. Bryan \\ National Center for \\ Veterans Studies \\ craig.bryan@utah.edu
}

\begin{abstract}
This paper describes the three phases of the Durkheim Project. For this project we developed a clinician's dashboard that displays output of models predicting suicide risk of veterans and active duty military personnel. During phase one, we built the clinician's dashboard and completed a Veterans Affairs (VA) predictive risk medical records study, based on an analysis of the narrative, or free text, portions of VA medical records, In phase two, we will predict suicide risk based on opt-in social media postings by patients using social media websites, e.g., Facebook. We describe the software infrastructure that we have completed for this phase two system. During phase three we will provide a three layer intervention strategy. We discuss our methodology for the three phases, including IRBapproved protocols for the first two phases and a soon-to-be approved IRB protocol for phase three.
\end{abstract}

\section{Introduction}

Diagnosis of psychological health and the prediction of negative events, such as suicide, or suicide ideation, is limited by: a) a lack of understanding of the true differentiating risks of suicidality (Health Promotion, 2010; Treating Soldiers, 2010) and b) a lack of near real-time reaction capability to large volumes of data. There is a need for broader coverage suicide risk detection and a better understanding of the expression of suicide ideation through data mining of text and images. The Durkheim Project's proposed solution is to provide continuous monitoring of text based information, such as found in social network user behavioral intent enabling intervention; facilitated by social / online data sources, powered by a medically-validated suicide risk classifier.

\section{Suicide risk and military culture}

The suicide rate among members of the United States Armed Forces has continued to rise for the past decade, beginning soon after the onset of military operations in Iraq and Afghanistan. Suicide is now the second-leading cause of death among military personnel, with more service members dying by suicide in 2012 than by combat-related causes (Zoroya, 2012). In response to steadily rising suicide rates among military personnel and veterans, researchers, clinicians, policy-makers, and military leaders have responded with an overwhelming and concerted effort to reverse these trends. Despite these considerable efforts, however, no evidence of effectiveness has been observed to date, resulting in considerable frustration for all involved. Although specific reasons explaining the lack of success to date are not yet known, it has been noted that most suicide prevention efforts used with military and veteran populations lack cultural relevance and do not incorporate several critical characteristics of the military culture that can create unique challenges from a suicide prevention perspective (Bryan et al., 2012). For instance, mental toughness and suppressive coping, fearlessness of death, and self-sacrifice are qualities that are valued in the military, but can serve as barriers to traditional prevention efforts.

The military culture values strength, resilience, courage, and personal sacrifice when faced with adversity. Weakness is not tolerated, and service members are expected to "shake it off" or "suck it up" when experiencing problems or illness. 
Suppression and avoidance have long been linked to mental health problems and emotional distress (Hayes et al., 1996), including suicidal ideation and suicide attempts (Najmi et al., 2007). Yet despite this "common sense" piece of knowledge, suppression and avoidance are nonetheless taught and reinforced within the military culture as a coping strategy because, in the short term after a stressful or traumatic event, suppression can actually reduce emotional distress and foster adaptation to extreme adversity (Beck et al., 2006; Bonanno 2004). This is especially relevant in combat situations, when natural grief responses may need to be suppressed to sustain adequate performance and achieve mission objectives. For example, crying in the midst of a fire fight is not adaptive or conducive to survival, and therefore must be stifled. Suppression and avoidance therefore presents the first paradox for understanding military and veteran suicide: a skill that is adaptive and useful in the short-term following a traumatic event can be detrimental and impair adaptive functioning in the long-term.

Military personnel are also explicitly trained to overcome their fear of injury and death, typically through repeated exposure to scenarios and environments that increasingly mimic actual combat situations, which habituates them to fear and eventually replaces this fear with exhilaration and/or other positive emotions (i.e., the opponent-process). Indeed, greater exposure to combat, especially combat marked by higher levels of violence and injury, are associated with less fear of death among military personnel (Bryan and Cukrowicz, 2011; Bryan et al. 2011). Fearlessness is an essential quality of a service member; retreating from danger and life-threatening situations are generally not conducive to an effective fighting force. Yet at the same time, fear of death is a well-known protective factor for suicide, given that individuals who are afraid to die are unlikely to attempt suicide, and fearlessness is associated with more severe levels of suicide risk among military personnel relative to civilian samples, and is associated with increased severity of suicide risk among military personnel (Bryan et al., 2010). Consequently, fearlessness about death paradoxically serves both as a necessary strength and asset for military personnel, yet also serves as a risk factor for suicide.

The military culture also places a premium on selflessness in the service of a higher good, and does not necessarily view life as the highest good in every situation. In the military, one's life might actually be viewed as subordinate to other, higher "goods" such as the well-being of others or ideals and principles such as freedom and justice. Laying down one's life for a greater good is widely considered to be one of the highest honors a service member can achieve. A considerable amount of research has converged on a very suicide-specific and dangerous thought process for suicidal behavior: perceived burdensomeness. Perceived burdensomeness entails the mistaken perception that "others would be better off without me" or that one's death is of greater value than one's life. Perceived burdensomeness and self-sacrifice are in many ways opposite sides of the same coin, and it is not yet clear how or when perceived burdensomeness ("taking" one's life) becomes mistaken for self-sacrifice ("giving" one's life) among military personnel and veterans.

These characteristics simultaneously function as an asset (in terms of military performance) and as a liability (in terms of suicide prevention) for military personnel and veterans, thereby creating a paradox for suicide prevention in military and veteran populations, and contributing directly to mental health stigma. Furthermore, the values of the military culture are generally at odds with the values and ideals of mental health systems, which value emotional vulnerability and helpseeking, and focus on deficiencies and clinical disorders, thereby reinforcing stigma even more. In essence, traditional prevention approaches have conceptualized suicide in a way that conflicts with the core identity and values of military personnel and veterans. To be effective, suicide prevention efforts must be culturally-relevant and integrate these values and ideals of military personnel and veterans.

\section{Related work}

In addition to the work related to military culture issues discussed in section 2, there are many linguistic approaches to analyzing suicide risk (Barak and Miron, 2005; Jones and Bennell, 2007; Lester, 2008a; Lester, 2008b; Lester, 2010a; Lester, 2010b; Lester et al., 2010; Lester and McSwain, 2010; Stirman and Pennebaker, 2001). In 2011, one of the Informatics for Integrating Biology \& the Bedside (i2b2) shared tasks was a sentiment analysis task to identify emotion in suicide notes (Combined Objective, 2011). Of this literature only Barak and Miron 
(2005) considers online text. Most other text analysis suicide research concerns analysis of suicide notes. There are studies of the writings of suicidal poets (Lester and McSwain, 2010; Stirman and Pennebaker, 2001) and studies involving distinguishing genuine and simulated suicide notes (Jones and Bennell, 2007; Lester, 2010a).

\section{The Durkheim Project}

\subsection{Overview}

The Durkheim Project consists of three phases. During the first phase, described in section 4.2, a clinician's dashboard was built and a Veterans Affairs (VA) predictive risk medical records study was completed, based on an analysis of the narrative, or free text, portions of VA medical records. Also during the first phase, the initial software infrastructure to collect and analyze the social media data for phase two, was designed and implemented. During the second phase, section 4.3, now underway, opt-in social media postings are being collected and will be analyzed. During the third phase, section 4.4, a pilot program will isolate serious suicide risk for individuals in real-time, and develop a prediction triage model for improved suicide intervention

\subsection{Phase 1: Veteran Affairs medical records study}

During phase 1 linguistics-driven prediction models were developed to estimate the risk of suicide. These models were generated from unstructured clinical notes taken from a national sample of United States VA medical records. The protocol for this study was approved by the Institutional Review Board (IRB) of the VA Medical Center, where the study was conducted. We created three matched cohorts: veterans who completed suicide, veterans who used mental health services and did not complete suicide, and veterans who did not use mental health services and did not complete suicide during the observation period ( $n=70$ in each group). From the clinical notes, we generated datasets of single keywords and multi-word phrases, and constructed prediction models using a supervised machinelearning algorithm based on a genetic programming framework, MOSES (Looks, 2006, 2007; Goertzel et al., 2013). MOSES can be described as a variant of a decision-tree forest, with certain genetic and maximum entropy techniques mixed in: maximum entropy to apply pressure to min- imize tree size and genetic to ensure tree species diversity. In our prior research we have found that MOSES consistently outperforms standard text classification approaches, such as Support Vector Machines (SVMs). The primary hyperparameter that we used was the dynamic feature size. The resulting inference accuracy was at first $65 \%$ and then consistently $67 \%$ or more. This was the prediction accuracy for assigning a patient to the correct cohort. These data suggest that computerized text analytics can be applied to unstructured sections of medical records to estimate the risk of suicide (Poulin et al. 2014). The resulting system could potentially allow clinicians to screen seemingly healthy patients at the primary care level, and to continuously evaluate suicide risk among psychiatric patients.

\subsection{Phase 2: Predicting risk with opt-in social media postings}

Although data collection and analysis for phase 2 is just beginning, the software development required for this data collection and analysis was completed during phase 1 . A phase 2 protocol for collecting and analyzing opt-in social media postings and presenting predictions to clinicians via the Durkheim Project's Clinicians' dashboard has also been approved by our IRB. When the system is fully operational, a clinician will see predictive models of suicide risk for a patient constructed from the patient's medical records and the patient's opt-in social media postings. Subjects are being recruited via targeted efforts. Subjects will be recruited through our collaboration with Facebook (PR Newswire 2013). A Facebook pop-up window will be used to recruit people that Facebook has identified as being military personnel or veterans.

\subsection{Phase 3: Intervention}

For phase 3, a protocol has been completed, which will soon be submitted to a final IRB. This protocol includes an unblinded, 3-cohort design, for a pilot program, which proposes to isolate serious suicide risks for individuals in real-time and to develop a prediction triage model for improved suicide intervention. Plans are to use and improve upon the linguistically-based prediction capabilities of the model developed during phase 1 . The phase 1 retrospective study was able to predict with limited accuracy before suicides occurred. The theoretic assumption is that wording chosen by those at risk will vary at different stages of risk. By building from ongoing observations from the phase 2 study and 
feedback obtained during the conduct of the phase 3 study, the aim is to adjust the linguisticsdriven model to predict suicide risk within the critical period for interventions of various levels of severity.

In this protocol, ongoing monitoring of the network will allow continuous updating and change in value of risk alert levels among the green-tored color coding. When the predictive system detects postings that indicate a certain threshold level of potential suicide risk, risk alerts are triggered in real-time and sent to either a monitoring clinician or a pre-identified buddy monitor, or to an automated system, which will generate supportive messages that are sent to the at-risk individual.

To better characterize the risk for the population of active-duty military and veterans, the analysis for this study will be limited to the primary participants. These primary participants may be newly recruited via the dedicated Facebook and mobile applications or, through that same dedicated application, from among those already participating in the phase 2 study. In either case, all primary participants must provide informed consent for this specific study. That is, those already involved in the phase 2 study must provide separate consent to participate in the phase 3 study. However, outside of the context of this study, the computerized intervention will be open to members of the general public who might wish to take advantage of the program's intervention potential. Primary participants are active duty U.S. military or veterans with English as a primary or secondary language, who agree to post to social media using English. The age limit for primary participants in the phase 3 study, as with phase 2 study, targets the age group most likely to actively use social media, i.e., those between the ages of 18 and 45.

\section{Results}

So far results are only available for the phase 1 study. For single-word models, the predictive accuracy was approximately 59\% (the average for 100 models), and scores for individual candidate models ranged from 46$67 \%$. Because our training sets are balanced, we have used accuracy as a surrogate for precision and recall. Accuracy was computed using five-way cross-validation. Models that used certain word pairs had significantly better scores than single-word models, though they are far less human readable. The phrases "negative assessment for PTSD" and "positive assessment for PTSD" carry different meanings. This phrase-based approach was more accurate than a single-word approach. For pre-selected word pairs, the individual model scores ranged from 52-69\%, with an average of $64 \%$ (for 100 models). In the final experiments, the combined Cohorts '1v2v3 classifier' had a peak performance of $70 \%$, and an average performance of $67 \%$.

\section{Discussion}

Our analyses were successful at determining useful text-based signals of suicide risk. We obtained accuracies of greater than $60 \%$ for ensemble averages of 100 models, and our individual model accuracies reached 67$69 \%$. Given the small size of the dataset and the fragmentary nature of the clinical notes, this performance level represents a significant achievement. For a classifier, these results represent a statistically significant 'signal'. Meanwhile, we showed that, methodologically, word pairs are more useful than single words for model construction on electronic medical record (EMR) data. Furthermore, the predictive feature words that distinguished each group were highly revealing, especially for the suicidal cohort, and were consistent with the existing medical literature on suicide. Many medical conditions have been associated with an increased risk for suicide, but these conditions have generally not been included in suicide risk assessment tools. These conditions include gastrointestinal conditions, cardiopulmonary conditions, oncologic conditions, and pain conditions. Also, some research has emerged that links care processes to suicide risk. The word "integrated" emerged as a key term in our study and is also reflected in the integrated care literature (Bauer et al., 2013).

Although the text on which our predictive model was based for the phase 1 medical records study was text written by a physician or other healthcare provider, our hypothesis 
is that some of the highly predictive features learned during phase 1 will carry over to the predictive modeling of opt-in social media postings during phase 2 . This text is written by the patient. However, we expect that some of the features, or concepts, will be the same due to the ability to do software based synonym matches Additionally, a physician or other healthcare worker may sometimes quote or paraphrase what a patient said when adding a note to the clinical record. A key predictive feature, such as the word "anxiety," may be used either by a clinician or a patient. We believe that the use of specialized text-analytic resources such as linguistic inquiry and word count (LIWC) would also help improve our results. Some preliminary results have been obtained using LIWC on our dataset.

In future research we plan to scale up the phase 1 medical records study from our current study where each cohort had 70 subjects to a study, using the same protocol, with at least 1000 subjects in each cohort. We also plan to transfer the predictive model built from the phase 1 study to the analysis of phase 2 opt-in social media postings. Once our phase 3 protocol has IRB approval, we plan to begin the phase 3 of the Durkheim Project, informed by the results, and ongoing follow-on research, of our phase 1 and 2 studies. In our future research we plan to use additional features from the structured portions of the medical record, as well as to use LIWC. In both our medical records and social media research we plan to use temporal analysis.

\section{Conclusion}

Although the phase 1 study was successful in distinguishing the cohort of completed suicides both from the control group cohort and the psychiatric cohort, it was difficult to distinguish text based noise from signal with high accuracy in our initial results. We expect that our planned follow-on study with 1000 subjects in each cohort will have much less problem in distinguishing signal from noise. Suicide risk prediction is a very diffi- cult problem. We believe that studies such as our phases 1 and 2 studies, which use supervised machine learning techniques, can uncover predictive risk factors that are not clearly understood by the medical community. At the same time, we also believe that more effective suicide risk prediction systems can be built based on the integration of machine learning methods and the expertise of suicidologists. In particular, building an understanding of military culture into our methods will be important.

\section{References}

Amy M. Bauer, Ya-Fen Chan, Hsiang Huang, Steven Vannoy, Jurgen Unützer. 2013. Characteristics, Management, and Depression Outcomes of Primary Care Patients Who Endorse Thoughts of Death or Suicide on the PHQ-9. J Gen Intern Med. Mar; 28(3):363-9. doi: 10.1007/s11606012-2194-2. Epub 2012 Aug 31.

Azy Barak, Ofra Miron. 2005. Writing Characteristics of Suicidal People on the Internet: A Psychological Investigation of Emerging Social Environments. Suicide and Life-Threatening Behavior 35(5) October.

Ben Goertzel, Nil Geisweiller, Pennachin, Cassio. 2013. Integrating Feature Selection into Program Learning. Proceedings of AGI-13, Springer. http://goertzel.org/agi-13/FS-MOSES_v1.pdf.

Chris Poulin, Brian Shiner, Paul Thompson, Linas Vepstas, Yinong Young-Xu, Benjamin Goertzel, Bradley Watts, Laura Flashman, Thomas McAllister. 2014. Predicting the Risk of Suicide by Analyzing the Text of Clinical Notes. PLOS ONE 9(1): e85733. doi:10.1371/journal.pone.0085733.

Combined Objective \& Subjective Shared Task Announcement: Call for Participation. 2011. https://www.i2b2.org/NLP/Coreference/Call.php.

Craig J. Bryan, Kelly C. Cukrowicz. 2011. Associations between types of combat violence and the acquired capability for suicide. Suicide and LifeThreatening Behavior, 41,126-136.

Craig J. Bryan, Kelly C. Cukrowicz, Christopher L. West, Chad E. Morrow. 2010. Combat experience and the acquired capability for suicide. Journal of Clinical Psychology, 66, 1044-1056.

Craig J. Bryan, Keith W. Jennings, David A. Jobes, John C. Bradley. 2012. Understanding and preventing military suicide. Archives of Suicide Research, 16, 95-110.

Craig J. Bryan, Chad E. Morrow, Michael D. Anestis, Thomas E. Joiner. 2010. A preliminary test of the 
interpersonal-psychological theory of suicidal behavior in a military sample. Personality and Individual Differences, 48, 347-350.

David Lester. 2008a. Computer Analysis of the Content of Suicide Notes from Men and Women. Psychological Reports, 102, 575-576.

David Lester. 2008b. Differences Between Genuine and Simulated Suicide Notes. Psychological Reports, 103, 527-528.

David Lester. 2010a. Linguistic Analysis of a Blog from a Murder-Suicide. Psychological Reports, 106(2): 342.

David Lester. 2010b. The Final Hours: A Linguistic Analysis of the Final Words of a Suicide. Psychological Reports, 106(3): 791-797.

David Lester, Janet Haines, Christopher Williams. 2010. Content Differences in Suicide Notes by Sex, Age, and Method: A Study of Australian Suicide Notes. Psychological Reports, 106(2): 475476.

David Lester, Stephanie McSwain. 2010. Poems by a Suicide: Sara Teasdale. Psychological Reports, 106(3): 811-812.

George Bonanno. 2004. Loss, trauma, and human resilience: Have we underestimated the human capacity to thrive after extremely aversive events? American Psychologist, 59, 20-28.

Gregg Zoroya. Army, Navy suicides at record high. 2012. USA Today. http://www.usatoday.com/story/news/nation/2012/ 11/18/navy-suicides-army/1702403/, November 18.

Health Promotion Risk Reduction Suicide Prevention. 2010. U.S. ARMY HP/RR/SP REPORT: http://usarmy.vo.llnwd.net/e1/HPRRSP/HP-RRSPReport2010_v00.pdf.

J. Gayle Beck, Berglind Gudmundsdottir, Sarah Palyo, Luana M. Miller, DeMond Grant. 2006. Rebound effects following deliberate thought suppression: does PTSD make a difference? Behavior Therapy, 37, 170-180.

LIWC. 2014. Linguistic Inquery and Word Count. http://www.liwc.net/ Accessed 28 April 2014.

Moshe Looks. 2006. Competent Program Evolution. $\mathrm{PhD}$ thesis, Washington University.

Moshe Looks. 2007. Meta-optimizing semantic evolutionary search. In: Lipson, H. (ed.), Genetic and Evolutionary Computation Conference, GECCO 2007, Proceedings, London, England, UK, July 711, p. 626.

Natalie J. Jones, Craig Bennell. 2007. The Development and Validation of Statistical Prediction Rules for Discriminating Between Genuine and Simulat- ed Suicide Notes. Archives of Suicide Research, 11:21-233.

PR Newswire. 2013

http://www.prnewswire.com/news-releases/thedurkheim-project-will-analyze-opt-in-data-fromveterans-social-media-and-mobile-content---seeking-real-time-predictive-analytics-for-suiciderisk-213922041.html Accessed 28 April 2014.

Sadia Najmi, Daniel M. Wegner, and Matthew K. Nock. 2007. Thought suppression and selfinjurious thoughts and behaviors. Behaviour Research and Therapy, 45, 1957-1965.

Shannon W. Stirman, James W. Pennebaker. 2001. Word Use in the Poetry of Suicidal and Nonsuicidal Poets. Psychosomatic Medicine 63:517-522.

Steven Hayes, Kelly G. Wilson, Elizabeth V. Gifford, Victoria M. Follette, and Kirk Strosahl. 1996. Experiential avoidance and behavioral disorders: A functional dimensional approach to diagnosis and treatment. Journal of Consulting and Clinical Psychology, 64, 1152-1168.

Treating Soldiers with Brain Injuries. 2010. Diane Rehm, NPR: June 24. 